Mens

Revue d'histoire intellectuelle et culturelle

mens

\title{
La danse pour tout un chacun : pratiques différenciées d'un loisir commercial à Montréal pendant l'entre-deux-guerres
}

\section{Peggy Roquigny}

Volume 16, numéro 1, automne 2015

Vie musicale amateur, populaire et américaine à Montréal, 1918-1958

URI : https://id.erudit.org/iderudit/1038983ar

DOI : https://doi.org/10.7202/1038983ar

Aller au sommaire du numéro

Éditeur(s)

Centre de recherche en civilisation canadienne-française

ISSN

1492-8647 (imprimé)

1927-9299 (numérique)

Découvrir la revue

Citer cet article

Roquigny, P. (2015). La danse pour tout un chacun : pratiques différenciées d'un loisir commercial à Montréal pendant l'entre-deux-guerres. Mens, 16(1), 21-61. https://doi.org/10.7202/1038983ar
Résumé de l'article

Pendant l'entre-deux-guerres, Montréal connaît un contexte favorable à l'épanouissement du marché du divertissement. La commercialisation du loisir, déjà enclenchée à la fin du XIX ${ }^{\mathrm{e}}$ siècle, s'amplifie malgré un contexte économique instable. Sur fond de musique jazz, la danse récréative n'échappe pas à ce mouvement de commercialisation. Les commerces proposant de s'adonner aux dernières danses à la mode se multiplient dans le centre-ville et rendent plus manifeste la popularité de l'activité dansante chez les citadins. Dans cet article, nous verrons que la commercialisation de la danse se décline en une variété de formules qui permettent des pratiques hétérogènes, associées à des formes de sociabilité variées, ce qui révèle des compréhensions différentes de la respectabilité. 


\title{
La danse pour tout un chacun : pratiques différenciées d'un loisir commercial à Montréal pendant l'entre-deux-guerres
}

\author{
Peggy Roquigny \\ Université du Québec à Montréal
}

\section{Résumé}

Pendant l'entre-deux-guerres, Montréal connaît un contexte favorable à l'épanouissement du marché du divertissement. La commercialisation du loisir, déjà enclenchée à la fin du XIX ${ }^{e}$ siècle, s'amplifie malgré un contexte économique instable. Sur fond de musique jazz, la danse récréative n'échappe pas à ce mouvement de commercialisation. Les commerces proposant de s'adonner aux dernières danses à la mode se multiplient dans le centre-ville et rendent plus manifeste la popularité de l'activité dansante chez les citadins. Dans cet article, nous verrons que la commercialisation de la danse se décline en une variété de formules qui permettent des pratiques hétérogènes, associées à des formes de sociabilité variées, ce qui révèle des compréhensions différentes de la respectabilité.

\section{Abstract}

During the interwar period, Montreal City is experiencing a suitable context for the development of the leisure market. The commercialization of entertaining activities, which had already started at the end of the nineteenth century, amplifies despite economic instability. Recreational dance on jazz rhythms belongs to this commercialization movement. In 
fact, the popularity of this activity among townspeople becomes obvious as dance businesses increase in the city centre. These businesses offer a variety of formulas that reveal as much as ways to enjoy the dancing commercialized entertainment; different ways that are associated with different forms of sociability and reveal different understandings of respectability.

Le loisir commercial prend sa place dans les grandes villes nordaméricaines dès le $\mathrm{xIx}^{\mathrm{e}}$ siècle et devient un élément incontournable de la vie urbaine de l'entre-deux-guerres. La danse que l'on pratique à titre d'amusement participe à ce processus de commercialisation $\mathrm{du}$ loisir. Les dernières décennies du XIX ${ }^{\mathrm{e}}$ siècle voient se multiplier les activités dansantes payantes lors d'événements organisés par des associations ou des clubs, par exemple. Le caractère lucratif de l'activité est formalisé par les écoles qui s'établissent progressivement. Après la Première Guerre mondiale, la commercialisation de la danse est un phénomène d'une intensité remarquable coïncidant avec la mode des musiques jazzées, lesquelles sont plus accessibles que jamais grâce à l'essor de la radiophonie et de l'industrie du disque ${ }^{1}$, et des danses modernes associées.

Aux États-Unis, cet engouement pour la pratique de la danse récréative dans des lieux commerciaux a suscité l'attention des chercheurs, notamment en ce qui concerne New York, Boston et Chicago. Lewis A. Erenberg et Burton W. Peretti ont consacré leurs travaux aux cabarets et nightclubs new-yorkais. À l'aide de journaux, de guides et de spicilèges, Erenberg se penche sur l'avènement des cabarets entre 1850 et 1930 et la façon dont l'élite les investit dans un contexte de remise en question de la culture victorienne. Peretti insiste plutôt sur le rapport entre les nightclubs et les gardiens de la morale incarnés

\footnotetext{
1 Nancy Marrelli, Stepping Out: The Golden Age of Montreal Night Clubs, 1925-1955, Montréal, Véhicule Press, 2004, p. 12 et 16 ; Sandria P. Bouliane et Peggy Roquigny, "Danser au son d'un orchestre dans le Montréal nocturne de l'entre-deux-guerres : salles de danse, gramophone et radio ", Intermédialités = Intermediality, $\mathrm{n}^{\circ} 26$ (automne 2015), [En ligne], [DOI : 10.7202/1037317ar].
} 
par les milieux politiques, policiers et réformateurs ${ }^{2}$. Kathy Lee Peiss et Leslie Gotfrit abordent les salles de danse comme des contextes particuliers permettant aux jeunes ouvrières d'expérimenter des rapports différents à leur genre, à leur corps, à leurs relations avec l'autre sexe et avec le pouvoir. Elles utilisent surtout des sources issues des mouvements réformateurs, rendant compte, du même coup, de la réception des pratiques de divertissement des jeunes travailleuses par ces milieux réformateurs bourgeois ${ }^{3}$. Les études de Randy McBee et de Susan Gedutis se distinguent notamment en valorisant le rôle qu'ont joué les salles de danse auprès des immigrants, lesquelles apparaissent comme des lieux d'intégration à la société et à la culture urbaine américaine tout en contribuant, directement ou indirectement, au renforcement des communautés ${ }^{4}$. Ces travaux ont en commun le fait de considérer les commerces spécialisés dans la danse sous l'angle des pratiques sociales et culturelles qui s'y expriment.

Au Canada, Montréal est, au même titre que Toronto, une métropole où le loisir commercial s'impose pendant l'entre-deuxguerres $^{5}$. Dans le paysage des centres-villes, les enseignes monumentales des cinémas et des théâtres en sont l'illustration la plus flagrante aux yeux du chaland ${ }^{6}$. Portant sur le monde du spectacle,

2 Lewis A. Erenberg, Steppin'Out: New York Nightlife and the Transformation of American Culture, 1890-1930, Westport, Greenwood Press, 1981; Burton W. Peretti, Nightclub City: Politics and Amusement in Manhattan, Philadelphie, University of Pennsylvania Press, 2007.

3 Kathy Lee Peiss, Cheap Amusements: Working Women and Leisure in the Turn-ofthe-Century New York, Philadelphie, Temple University Press, 1986; Leslie Gotfrit, Dancing Back in the Jazz Age: Discourses of Danger and Possibilities of Pleasure, mémoire de maîtrise (éducation), Toronto, Université de Toronto, 1992.

${ }^{4}$ Randy McBee, Dance Hall Days: Intimacy and Leisure among Working-Class Immigrants in the United States, New York, New York University Press, 2000; Susan Gedutis, See you at the Hall: Boston's Golden Era of Irish Music and Dance, Boston, Northeastern University Press, 2004.

5 Paul-André Linteau, La rue Sainte-Catherine : au cour de la vie montréalaise, Montréal, Musée Pointe-à-Callière, Les Éditions de l'Homme, 2010, p. 127-161.

6 Paul S. Moore, "Movie Palaces on Canadian Downtown Main Streets ", Revue d'histoire urbaine $=$ Urban History Review, vol. 32, n 2 (printemps 2004), p. 3-20. 
ses musiciens et ses artistes, les travaux de John Gilmore, de Nancy Marrelli, d'André G. Bourassa et Jean-Marc Larrue nous montrent que les Montréalais avaient accès à des nightclubs et à des cabarets ${ }^{7}$. Certains sont répertoriés et quelques pistes sont fournies sur les types de clientèles, mais les pratiques des clients ne sont guère examinées. Ce sont plutôt des travaux relatifs à l'histoire des femmes qui les abordent, considérant le rôle de la danse dans la "sociabilité de jeunesse $^{8}$ ", un rôle qui varie selon le milieu social et culturel de la jeune fille et selon le type de lieu qu'elle fréquente 9 . Plusieurs historiennes mettent en évidence le comportement des femmes en regard des discours normatifs, voire coercitifs dans lesquels on dénonçait les attitudes considérées inappropriées, immorales, voire dangereuses, de façon à obtenir un réajustement de ces comportements, que ce soit par la sensibilisation, la réforme ou l'action judiciaire ${ }^{10}$. Selon certaines sources et par l'attention accordée à la façon dont les femmes négocient avec les discours normatifs auxquels elles font face, ces

${ }^{7}$ John Gilmore, Swinging in Paradise: The Story of Jazz in Montreal, Montréal, Véhicule Press, 1988; André-G. Bourassa et Jean-Marc Larrue, Les nuits de la "Main": cent ans de spectacles sur le boulevard Saint-Laurent (1891-1991), Montréal, VLB éditeur, 1993, p. 115-127, 133, note 174; Marrelli, Stepping Out.

${ }^{8}$ Denise Lemieux et Lucie Mercier, Les femmes au tournant du siècle, 1880-1940: âges de la vie, maternité et quotidien, Québec, Institut québécois de recherche sur la culture, 1989 , p. 8.

9 Ibid., p. 109-121; Denyse Baillargeon, Ménagères au temps de la Crise, Montréal, Éditions du remue-ménage, 1993, p. 74-75; Denise Girard, Mariage et classes sociales: les Montréalais francophones entre les deux guerres, Sainte-Foy, Institut québécois de recherche sur la culture, 2000, p. 33-74; Magda Fahrni et Yves Frenette, " "Don't I long for Montreal” : l'identité hybride d'une jeune migrante franco-américaine pendant la Première Guerre mondiale ", Histoire sociale = Social History, vol. 41, n 81 (mai 2008), p. 90.

10 Tamara Myers, Caught: Montreal's Modern Girls and the Law, 1869-1945, Toronto, University of Toronto Press, 2006; Patricia Comtois, Jeunes femmes et loisirs commerciaux durant les Années folles (1919-1929) : étude des discours ecclésiastiques et journalistiques, mémoire de maîtrise (histoire), Montréal, Université du Québec à Montréal, 2007, p. 79-106; voir aussi Carolyn Strange, Toronto’s Girl Problem: The Perils and Pleasures of the City, 1880-1930, Toronto, University Press of Toronto, 1995. 
analyses rejoignent celles de Kathy Lee Peiss sans toutefois porter la même attention aux commerces spécialisés dans la danse eux-mêmes.

Cet article, tout comme la thèse dont il découle ${ }^{11}$, propose de lier l'étude des lieux commerciaux montréalais non pas à l'histoire du spectacle mais à celle des pratiques qu'ils suscitent. Ce travail s'inscrit dans la lignée des travaux d'Erenberg et de Peiss, car il aborde la vie nocturne " as reflecting not the pathology of urban culture [...] but its positive expression, its unique identity ${ }^{12}$ " et porte autant intérêt aux salles de danse qu'aux cabarets. Effectivement, en étudiant les pratiques qui peuvent être associées aux divers types de commerces spécialisés dans la danse, restaurants dansants, cabarets et salles de danse, cet article souhaite montrer que la variété de l'offre commerciale montréalaise permet de satisfaire les attentes multiples en matière de divertissement dansant, car ces cadres variés autorisent l'expression de différentes formes de sociabilité étroitement liées à des compréhensions également différentes de la notion de respectabilité. Si l'offre est modelée par les entreprises, la liberté des clients, qui, nonobstant leurs milieux et leurs moyens, peuvent explorer les commerces dans toute la ville, doit être prise en considération.

\section{Sources et méthode}

L'analyse s'appuie sur un corpus de cent-vingt-six commerces ayant opéré à Montréal entre 1919 et 1940, repérés grâce aux annuaires Lovell et aux publicités contenues dans les journaux et les programmes de spectacles $^{13}$. Ces sources ont permis de déterminer, pour

11 Peggy Roquigny, Les plaisirs de la danse à Montréal : transformation d'un divertissement et de ses pratiques, 1870-1940, thèse de doctorat (histoire), Montréal, Université du Québec à Montréal, 2012.

12 Howard P. Segal, "Compte rendu de Erenberg, Lewis, A., Steppin'Out: New York Nightlife and the Transformation of American Culture, 1890-1930", Urban History Review $=$ Revue d'histoire urbaine, vol. 12, n 2 (octobre 1983), p. 151.

13 John Lovell (dir.), Lovell's Montreal Directory, Montréal, 1918-1940. Le dépouillement des journaux concerne essentiellement le Montreal Daily Star et La Presse, et s'appuie sur les précieuses archives d'Iro Valaskakis-Tembeck et sur les travaux de dépouillement effectués par Alex Robertson. Voir Bibliothèque de la danse Vincent- 
chaque commerce, son type (salle de danse, cabaret, restaurant dansant, changements éventuels de fonction), sa localisation, ses années d'ouverture et de fermeture à l'année près ${ }^{14}$. Combinées à divers témoignages que nous présenterons ci-dessous, les publicités, leur iconographie notamment, ont permis de saisir les visées des établissements en matière de clientèle et leur arrimage éventuel à l'identité sociale et culturelle du quartier dans lequel ils opèrent (voir le tableau 1). Effectivement, le centre-ville (de Guy, à l'ouest, à Amherst, et de Sherbrooke à Notre-Dame et Craig [actuellement Saint-Antoine]), qui concentre la majorité des commerces repérés, se divise a priori en trois secteurs aux identités sociales et culturelles fortes. Saint-Antoine Nord (SAN), délimité par les rues de la Montagne, Burnside (actuellement de Maisonneuve), Mansfield et Dorchester (actuellement René-Lévesque), est étroitement lié aux activités de la bourgeoisie montréalaise, et particulièrement anglophone. Même si les zones résidentielles de ce secteur se sont déjà largement déplacées vers Westmount ${ }^{15}$, les demeures luxueuses qui subsistent dans le mile carré, les grands commerces comme Goodwin's, Ogilvy et Morgan, les grands hôtels Windsor, RitzCarlton et Mont-Royal ${ }^{16}$, ainsi que les nombreux théâtres comme le

\footnotetext{
Warren (ci-après BVW), Fonds Iro Valaskakis-Tembeck (ci-après, Fonds IVT), P101; Collections spéciales, Bibliothèques, Université Concordia (ci-après CSC), Fonds Alex Robertson, P023. Remarque : les auteurs et les pages des articles ou des publicités ne sont pas précisés dans la plupart de ces archives et spicilèges. C'est la raison pour laquelle nous ne les mentionnons pas dans les références ci-après.

14 Les annuaires Lovell étaient alors publiés à cheval sur deux années, par exemple : 1918-1919.

15 Paul-André Linteau, Histoire de Montréal depuis la Confédération, Montréal, Éditions du Boréal, 1992, p. 195-196, 354; Harold Bérubé, Des banlieues qui se distinguent : gouverner Westmount, Pointe-Claire et Mont-Royal (1880-1939), thèse de doctorat (études urbaines), Montréal, INRS, 2008, p. 74-79.

16 Michèle Benoît et Roger Gratton, Pignon sur rue : les quartiers de Montréal, Montréal, Guérin éditeur, 1991, p. 65-67; Linteau, Histoire de Montréal, p. 34, 79-80, 107; Adrian Waller, No Ordinary Hotel: The Ritz-Carlton First Seventy-Five Years, Montréal, Véhicule Press, 1989; Isabelle Gournay, « Prestige et compétence des architectes américains " et "Manifestations du gigantisme au centre-ville ", dans Isabelle Gournay et France Vanlaethem (dir.), Montréal métropole, 1880-
} 


\section{Tableau 1}

Nombre d'établissements par type et par secteur, entre 1918 et 1940

\begin{tabular}{|l|c|c|c|c|c|}
\hline $\begin{array}{l}\text { Secteur/Types } \\
\text { d'établissements }\end{array}$ & $\begin{array}{c}\text { Saint- } \\
\text { Antoine } \\
\text { Nord }\end{array}$ & $\begin{array}{c}\text { Saint- } \\
\text { Antoine } \\
\text { Sud }\end{array}$ & $\begin{array}{c}\text { Centre- } \\
\text { ville Est }\end{array}$ & $\begin{array}{c}\text { Hors } \\
\text { centre- } \\
\text { ville }\end{array}$ & Total \\
\hline $\begin{array}{l}\text { Restaurants } \\
\text { dansants }\end{array}$ & 16 & 1 & 7 & 4 & 28 \\
\hline Cabarets & 23 & 8 & 11 & 3 & 45 \\
\hline Salles de danse & 4 & 1 & 8 & 28 & 41 \\
\hline $\begin{array}{l}\text { Salles de danse } \\
\text { dans écoles }\end{array}$ & 4 & 0 & 0 & 2 & 6 \\
\hline $\begin{array}{l}\text { Établissements à } \\
\text { fonction changeante }\end{array}$ & 3 & 0 & 2 & 1 & 6 \\
\hline $\begin{array}{l}\text { Nbre total } \\
\text { d'établissements }\end{array}$ & 50 & 10 & 28 & 38 & 126 \\
\hline
\end{tabular}

His Majesty's, le Loews, le Palace, le Princess, l'Imperial, l'Orpheum et le Capitol ${ }^{17}$ en sont autant de symboles. Saint-Antoine Sud (SAS), délimité par les rues de la Montagne, Osborne (de La Gauchetière), Mansfield et Saint-Jacques, est, par contraste, un secteur ouvrier et industrie ${ }^{18}$ où la communauté noire est implantée depuis la fin du $\mathrm{XIX}^{\mathrm{e}}$ siècle $^{19}$. Quant au centre-ville Est, il accueille des commerces spécialisés dans la danse entre Bleury, Sherbrooke, Amherst et Craig. Cette zone géographique, aux caractéristiques des plus diversifiées, comporte, dans sa section sud-ouest, le district Dufferin (entre Bleury, Sainte-Catherine, Saint-Laurent et Craig). Il s'agit d'un milieu populaire, prolétaire, industriel et commercial francophone,

1930, Montréal, Centre Canadien d'Architecture (CCA) et Éditions du Boréal, 1998, p. 127-145, p. 169-199.

17 Current Events, 26 octobre 1928, p. 14-15.

18 Linteau, Histoire de Montréal, p. 80.

19 Dorothy W. Williams, Les Noirs à Montréal, 1628-1986 : essai de démographie urbaine, trad. de Pierre DesRuisseaux, Montréal, VLB éditeur, 1998. 
mais aussi particulièrement multiethnique, car bien placé pour accueillir les nouveaux arrivants. On y relève plus de vingt ethnies en $1928^{20}$. Ce district chevauche la partie sud-ouest du Red Light, qui s'étend à travers l'est du centre-ville jusqu'à Sherbrooke et SaintDenis. Le Red Light, dont Saint-Laurent et Sainte-Catherine sont les fils conducteurs, dissimule un monde interlope où prostitution, corruption, jeu, stupéfiants et divertissements populaires se croisent pour satisfaire une clientèle hétéroclite - ou profiter d'elle ${ }^{21}$. Par ailleurs, une population étudiante gravite autour de Saint-Denis et de Sainte-Catherine, et les rues Sherbrooke et Saint-Denis abritent encore une partie de la bourgeoisie locale francophone même si elle a entamé sa migration vers Outremont ${ }^{22}$. Hors du centre-ville, on trouve aussi des commerces spécialisés dans la danse, surtout des salles, dont nous savons cependant peu de choses.

Des tendances nettes se dégagent des trois quartiers fortement caractérisés du centre-ville : bien des établissements proposent des tarifs et des formules susceptibles de correspondre, d'abord et avant tout, au profil socioculturel des habitants du secteur, contribuant du même coup à l'identité sociale et culturelle des quartiers dans lesquels ils se situent. Néanmoins, les commerces peuvent offrir une formule différente de celle couramment proposée dans un quartier pour les habitants du secteur. De même, la mobilité des individus vient brouiller les cartes : on ne peut prétendre que l'environnement immédiat, le voisinage, le quartier, constitue le bassin exclusif de la clientèle d'un établissement, que cela soit ou non son objectif.

20 Percy A. Robert, Dufferin District: An Area in Transition, mémoire de maîtrise (sociologie), Montréal, Université McGill, 1928.

21 Andrée Lévesque, La norme et les déviantes: les femmes au Québec pendant l'entredeux-guerres, Montréal, Éditions du remue-ménage, 1989, p. 71 ; Daniel Proulx, Le Red Light de Montréal, Montréal, VLB éditeur, 1997; Bourassa et Larrue, Les nuits de la "Main ", p. 14-15.

22 Benoît et Gratton, Pignon sur rue, p. 36-37; Linteau, Histoire de Montréal, p. $188-191,195-196,354$. 
Nous examinerons d'abord la variété des formules proposées par les restaurants dansants et les cabarets (que nous traitons simultanément étant donné leurs liens étroits), aussi bien en ce qui concerne la politique d'accès et la sociabilité dansante, ce qui nous amène à distinguer les lieux favorisant les formes de sociabilité formelle de ceux acceptant les pratiques plus informelles. Nous terminerons avec les salles de danse, dont la fonction sociale, fondamentalement différente de celle des cabarets et des restaurants dansants, influe directement sur la clientèle et la sociabilité qui sont, néanmoins, également variées.

\section{Restaurants dansants et cabarets au service de la bienséance}

Au sortir de la Première Guerre mondiale, les restaurants dansants sont, avec les salles de danse, la principale forme de commerce à offrir l'activité dansante. La formule qui associe la table à une piste de danse avec orchestre est suffisamment appréciée pour que le nombre de restaurants dansants augmente régulièrement jusqu'à atteindre un maximum en 1927-1928. Le déclin qui s'enclenche alors, avant même le début de la crise économique, est constant et prononcé tout au long des années 1930. Certes, la crise économique n'est pas un contexte favorable au redressement du nombre des restaurants dansants. Mais c'est plutôt dans l'intérêt croissant pour la formule des cabarets qu'il faut chercher la cause du déclin des premiers à la faveur des seconds. De fait, les cabarets, eux, connaissent une croissance remarquable et ininterrompue jusqu'en 1934, dépassant les restaurants dansants dès 1931. La prohibition en vigueur aux États-Unis entre 1919 et 1933 a pu contribuer à retarder l'influence de la crise économique sur le développement de certains commerces liés au divertissement, notamment avec la venue d'artistes newyorkais et de touristes américains ${ }^{23}$. Et bien que la crise économique

23 Bourassa et Larrue, Les nuits de la " Main », p. 118-119. 
de 1929 soit à son paroxysme à Montréal en $1933^{24}$, c'est surtout à partir de 1936 qu'elle touche plus durement les commerces spécialisés dans la danse ${ }^{25}$. Malgré tout, le succès des cabarets est irréversible et s'obtient au détriment des restaurants dansants. Dans le Montreal Daily Star, un article évoquant l'ouverture du Frolic's Café en 1930 explique ainsi le succès croissant des cabarets :

Montreal has never been overland [sic] with good cabarets. Yet the cabaret is becoming an increasingly popular form of entertainment-a place where people can gather formally or informally, chat, dine or sup, dance and watch dancers, singers and comedians between the dances - not from a theatre seat but from the pleasantly intimate table beside the dancing floor ${ }^{26}$.

Dans ce texte, on insiste sur la panoplie d'activités de détente que permettent les cabarets, ajoutant les spectacles au repas, à l'orchestre et à la danse, sans que les tarifs soient plus élevés pour autant. Les restaurants dansants ne résistent pas à ce cheval de Troie et plusieurs se convertissent à la formule cabaret afin d'assurer leur survie. En 1940 , les seuls restaurants dansants qui subsistent comme tels sont des commerces qui tirent également des bénéfices d'autres fonctions. Le Venus Sweets est un confiseur ${ }^{27}$; les autres sont des hôtels bien établis : le Windsor, le Ritz-Carlton, le Mont-Royal, le Queen's et le LaSalle. La citation mentionne également la diversité de comportements que permettent les cabarets : " a place where people can gather

${ }^{24}$ Linteau, Histoire de Montréal, p. 283-284.

25 Roquigny, Les plaisirs de la danse à Montréal, p. 269-276.

${ }_{26}$ Montreal Daily Star, 4 janvier 1930, BVW, Fonds IVT, P101.

27 Orpheum, 16 septembre 1923, Bibliothèque et Archives nationales du Québec (ci-après BAnQ), Collection programmes de théâtre; Capitol Entertainment, 15 avril 1922, Archives de la Ville de Montréal (ci-après AVM), Fonds Ægidius Fauteux, série 11, Programmes de spectacles, dossier BM001-11-D27. Remarque : les collections programmes de théâtre et de danse de BAnQ sont en cours d'intégration à Collection patrimoniale de programmes de spectacles, actuellement en réorganisation. Voir le site de BAnQ : [http://www.banq. qc.ca/collections/collections_patrimoniales/collections_speciales/programmes_ spectacles/index.html\#C]. 
formally or informally ». Cela nous amène considérer la variété des formules proposées par les restaurants dansants (nous les incluons) et les cabarets; l'emplacement de l'établissement dans la ville contribue à déterminer sa formule et sa clientèle, mais d'autres facteurs sont aussi à considérer.

\section{Une respectabilité toute bourgeoise?}

Un client ou une cliente ${ }^{28}$ qui souhaite se divertir dans un établissement où la sociabilité formelle est privilégiée trouvera de quoi satisfaire ses attentes en se tournant vers des établissements destinés à la bourgeoisie.

Dès le tournant du siècle, les commerces spécialisés dans la danse visant une clientèle bourgeoise mixte ont dû faire la preuve qu'ils étaient en mesure de se conformer aux exigences de cette clientèle en matière de respectabilité2 ${ }^{29}$. Cet enjeu concerne surtout les femmes ${ }^{30}$, que les « anciens modèles [victoriens] de féminité définis par la chasteté et la modestie ${ }^{31}$ " de la seconde moitié du $\mathrm{Xx}^{\mathrm{e}}$ siècle cantonnaient à la sphère privée ou associative ${ }^{32}$. De fait, pour remplir cette obligation d'être et de paraître moralement et physiquement vertueuse, irréprochable, autrement dit respectable, on soigne l'apparence, on contrôle le comportement et les circonstances engageant des relations sociales afin d'éviter toute ambiguïté, toute situation équivoque ${ }^{33}$.

${ }^{28}$ Le genre masculin sera, pour la suite du texte, utilisé à titre générique. De façon à alléger le propos, il représentera aussi bien des clients que des clientes.

29 Se référer à Erenberg, Steppin'Out, qui l'a très bien montré pour New York (voir notamment p. 77 et 114 ).

30 Ibid., p. 18.

31 Denyse Baillargeon, Brève histoire des femmes au Québec, Montréal, Éditions du Boréal, 2012, p. 124-125.

32 Margaret Westley, Grandeur et déclin : l'élite anglo-protestante de Montréal, 19001950, Montréal, Libre Expression, 1990, p. 161-168, 176-178, 219-251 ; Linteau, Histoire de Montréal, p. 170, 173-174; Lemieux et Mercier, Les femmes au tournant du siècle, 1880-1940, p. 314-316; Collectif Clio, L'histoire des femmes au Québec depuis quatre siècles, Montréal, Le Jour, 1992, p. 279-280.

33 Karen Halttunen, Confidence Men and Painted Women: A Study of Middle-Class Culture in America, 1830-1870, New Haven, Yale University Press, 1982. 
Bref, la respectabilité, telle qu'entendue par la bourgeoisie et par la classe moyenne de tradition victorienne, est soutenue par une sociabilité formelle, par le fait de bien se tenir ${ }^{34}$. Lorsqu'il s'agit de divertissement dansant, lequel entraîne souvent des relations avec l'autre sexe, la respectabilité impose d'éviter toute situation de séduction ou d'apparence de séduction indésirable. Comme le note Denyse Baillargeon, certaines femmes des années 1920 sont séduites par des modèles de féminité (l'archétype de la flapper et de la sportive) qui apparaissent comme une solution de rechange aux " anciens modèles " victoriens. Mais d'autres préfèrent ne pas les remettre en question de façon radicale, voire s'y opposent ${ }^{35}$. Les commerces qui souhaitent attirer cette clientèle doivent donc la convaincre qu'ils sont un lieu public dans lequel les femmes peuvent intégrer la société de divertissement moderne sans remettre en cause leur respectabilité. Ce sont donc des établissements qui, dans leurs publicités, valorisent des attitudes de sociabilité formelle, bienséantes.

Les commerces visant une clientèle bourgeoise sont facilement repérables, ne serait-ce qu'en raison des tarifs qu'ils pratiquent, les membres de la bourgeoisie appréciant un train de vie et un luxe proportionnels à leurs moyens. Celui qui recherche un établissement qui remplisse ces exigences en matière de luxe et de bienséance trouvera facilement satisfaction dans Saint-Antoine $\operatorname{Nord}^{36}$ puisque ce secteur est investi par la bourgeoisie anglo-montréalaise. À proximité des théâtres et des grands hôtels, ou à l'intérieur même de ces établissements, se situent la plupart des restaurants dansants et des cabarets aux tarifs les plus élevés : entre un et deux dollars pour les tables d'hôte et dîners de début de soirée. Quant aux soupers de fin de soirée, qui permettent de se restaurer après une sortie au théâtre par

34 Erenberg, Steppin'Out, p. 5-21; Halttunen, Confidence Men and Painted Women.

35 Baillargeon, Brève histoire des femmes au Québec, p. 123-124.

36 Pour une étude axée sur l'exclusivisme des loisirs dansants de la bourgeoisie anglo-montréalaise, voir Peggy Roquigny, "Loisirs dansants de la bourgeoisie anglo-montréalaise : transformation et persistance des lieux de pratique, 18701940 ", Urban History Review = Revue d'histoire urbaine, vol. 40, $\mathrm{n}^{\circ} 1$ (automne 2011), p. 17-29. 
exemple, ils sont à la carte et les prix ne sont pas précisés. Quelques commerces, parmi ceux les plus réputés, ajoutent à cela des frais de couvert de un dollar. On nommera, au premier rang, les restaurants dansants des grands hôtels, le Windsor, le Mont-Royal, mais aussi le Venetian Gardens (1918-1932, Sainte-Catherine Ouest) ${ }^{37}$, le Cosy Grill (1922-1934, Sainte-Catherine Ouest) et le Ritz-Carlton (19121940, Sherbrooke Ouest). En annonçant de tels prix, ces établissements ciblent une clientèle aisée, voire la restreignent aux plus nantis.

Si la majorité des commerces visant une clientèle bourgeoise se situe dans Saint-Antoine Nord, le client qui préfère Saint-Antoine Sud ou l'est du centre-ville peut également y trouver satisfaction. Le Queen's Hotel (1893-1940+ ${ }^{38}$, Windsor, entre Saint-Jacques et SaintAntoine, SAS), faisant valoir dans son iconographie publicitaire un milieu très distingué, propose le dîner dansant à un dollar cinquante. Pour le souper, il faut prévoir des frais de couvert de cinquante cents ${ }^{39}$. Au Matou botté (1929-1932, Saint-Denis, centre-ville Est) on propose les dîners dansants à un dollar de $19 \mathrm{~h}$ à $21 \mathrm{~h}^{40}$. Quant au restaurant dansant de l'hôtel Place Viger (1898-1935, Craig Est, centre-ville Est), qui peut compter sur la bourgeoisie locale, les touristes ou les gens d'affaires qui se rendent à la gare Viger ${ }^{41}$, il propose le souper dansant du samedi soir à deux dollars en 1930, puis à un dollar cinquante en 1931, sans frais pour le couvert ${ }^{42}$.

Il existe cependant des commerces visant une clientèle plus modeste mais néanmoins soucieuse de se comporter conformément

37 Ibid.

38 Cette mention $1940+$ et les suivantes signifient que l'année de fermeture du commerce est ultérieure à la période étudiée, soit après 1940 .

39 Current Events, 16 juin 1928, p. 10-11.

40 La Presse, 26 septembre 1932, BVW, Fonds IVT, P101.

41 Julie Duchesne, Terminus ferroviaires et espace urbain : le Canadien Pacifique et la transformation de l'est de Montréal, 1870-1915, mémoire de maîtrise (histoire), Montréal, Université du Québec à Montréal, 2003, p. 148-152.

42 The Gazette, 10 octobre 1930, p. 6, BVW, Fonds IVT, P101; Montreal Daily Star, 9 décembre 1931, p. 14; La Presse, 19 novembre 1930, p. 8, et 18 novembre 1931 , p. 8. 
aux attentes des élites qu'elle cherche à rejoindre par la mobilité sociale - en l'occurrence, la classe moyenne. Dans ces cas, la tarification plus accessible (autour de quarante cents ${ }^{43}$ ) ne permet pas de rattacher le commerce à cette respectabilité bourgeoise de façon aussi évidente. On s'assure donc de mettre en valeur, dans les publicités, le bon goût qui caractérise le commerce et, implicitement, les comportements tout à fait convenables qui s'y expriment. Ces commerces se situent particulièrement dans ce secteur socialement et culturellement hétérogène qu'est le centre-ville Est, ce qui indique que les cheap amusements de Saint-Laurent et les bordels du Red Light ne sont pas les seules options pour se divertir à l'est de Bleury. Ainsi, le Moonlight Café (1930-1933, Amherst) valorise à la fois ses prix abordables et la qualité de l'établissement dans une publicité dont le caractère bilingue révèle le désir d'attirer les clientèles anglophone et francophone :

After the show is over, visit the Moonlight Garden Cafe (corner of Amherst and St. Catherine East).

Dîner au Moonlight Cafe [...] Le restaurant le plus moderne en ville, décoré avec goût, où l'on mange tout à son aise. Repas régulier tous les jours, 35 c. et plus. Service à la carte à toute heure à prix très raisonnable. Danse tous les soirs depuis 9 heures. Couvert gratis. Souper dansant, 6 h 30 à 7 h 30.7 musiciens [Ce paragraphe est répété en anglais dans la publicité].

\section{High class food and Oriental Dishes a speciality ${ }^{44}$.}

De même, le Café Impérial (1917-1932, Sainte-Élisabeth), où l'on danse tous les soirs, sert le repas normal à trente-cinq cents et plus, ou à la carte, et le repas spécial avec alcool pour quarante-cinq cents en 1928 et $1930^{45}$, dans un cadre simple mais soigné et joliment

43 Nous possédons de la documentation concernant 26 des 29 établissements du centre-ville Est, et 8 d'entre eux indiquent leurs tarifs dans leurs publicités.

44 Théatre National, du 3 mars au 31 mai 1930, BAnQ, Collection programmes de théâtre.

45 La revue du théâtre, 2 décembre 1928, AVM, Fonds Ægidius Fauteux, série 11, 
orné de plantes et d'alcôves ${ }^{46}$. Pour sa part, le Amherst Garden (19261929, Sainte-Catherine Est), " le meilleur restaurant de l'est ", alterne tarifs abordables en semaine (des minimums à cinquante cents) et tarifs plus élevés (un dollar) les samedis et jours de fête. On va même jusqu'à deux dollars pour la soirée du réveillon du jour de l'An, lors de laquelle on peut danser au son de l'orchestre R. E. Demers. Le couple attablé qui illustre les annonces publicitaires ne laisse planer aucun doute sur la sobriété de l'établissement et la condition à la fois respectable et plus modeste des clients qu'il s'attend à recevoir.

Le client a donc accès à des cabarets ou des restaurants dansants qui misent sur la respectabilité telle que comprise par les milieux bourgeois de tradition victorienne et il peut choisir le lieu qu'il fréquente en fonction de ses moyens. Quant au quartier, si la majorité des commerces de luxe se situent dans Saint-Antoine Nord et que les plus abordables se trouvent plutôt dans le centre-ville Est, la règle n'est pas absolue ${ }^{47}$.

\section{Respectabilité dans l'activité dansante}

Si le secteur, les prix ou la façon dont sont tenus les établissements sont autant d'indices qui, utilisés séparément ou conjointement, peuvent aider le client à évaluer les normes de respectabilité proposées par un commerce, il peut également se fier aux attitudes des danseurs représentées dans l'iconographie publicitaire. À l'instar du Amherst Garden, les publicités des commerces mentionnés jusqu'ici misent sur le couple et les groupes préétablis ${ }^{48}$. En décourageant implicitement ceux qui souhaiteraient s'y rendre seuls, on exprime le refus de

Programmes de spectacles, dossier BM001-11-D33 (évoque les deux tarifs); Théâtre National, du 7 juin au 28 juillet 1930, BAnQ, Collection programmes de théâtre (évoque seulement le tarif à 35 cents).

46 Publicité avec photographie du Café impérial, Théâtre National, du 2 au 16 septembre 1929, BAnQ, Collection programmes de théâtre.

47 On compte également quelques commerces moins dispendieux dans Saint-Antoine Nord, avec des tarifs à partir de 75 cents.

48 Roquigny, Les plaisirs de la danse à Montréal, p. 347-356. 

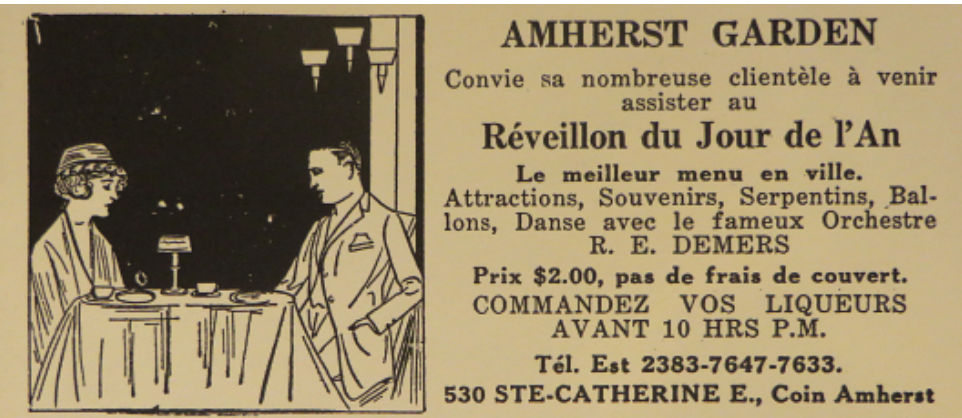

Figure 1 - Théatre Canadien Français, 26 décembre 1926 et 23 mars 1927 (AVM, Fonds Ægidius Fauteux, série 11, Programmes de spectacles, dossiers BM001-11-D31 et D32, [En ligne], [https://archivesdemontreal.ica-atom.org/ programmes-de-spectacles-1926]).

toute dynamique axée sur la rencontre, la séduction ouverte ou le commerce sexuel, du moins à l'intérieur de l'établissement. On se distingue ainsi d'autres lieux qui marquent la vie nocturne montréalaise, comme le 92 rue Cadieux, situé dans le Red Light, cité dans le rapport de la commission Coderre sur la prostitution comme "le bordel le plus populaire de l'après-guerre, avec ses trois salles de danse, son joueur de piano ${ }^{49} "$.

Cela n'empêche pas le client de concilier cette respectabilité conforme aux standards bourgeois avec la pratique de danses à la mode. À cette fin, les commerces engagent des orchestres pour des périodes plus ou moins longues. Ainsi, dans les années 1930, à l'hôtel Mont-Royal, avec Charles Dornberger, ses musiciens et leur « twinkling tunes ", les clients peuvent s'adonner au tango, si l'on en croit l'image qui accompagne la publicité de $1935^{50}$. Myron Sutton établit une

${ }^{49}$ Lévesque, La norme et les déviantes, p. 197. Ce lieu ne fait pas partie de notre répertoire car il n'apparaît pas (et pour cause) dans les sources que nous avons sélectionnées.

50 Capitol Entertainment, 15 avril 1922, AVM, Fonds Ægidius Fauteux, série 11, Programmes de spectacles, dossier BM001-11-D28; Orpheum Theatre, 3 décembre 1923, Ibid., dossier BM001-11-D28; La Presse, 10 novembre 1924; Current Events, 26 octobre 1928; pour Dornberger : Théâtre Impérial, $1^{\text {er }}$ décembre 1933, 
distinction entre la musique qui se joue dans les restaurants dansants des grands hôtels et celle exécutée par son orchestre de swing, les Canadian Ambassadors: "Uptown in the big hotels they had a different type of music than we had [swing]. They had ballroom music ${ }^{51}$. "Dans ces établissements de Saint-Antoine Nord, Uptown, les musiques modernes, jazzées ou latines y sont exécutées à des rythmes compatibles avec des mouvements et des attitudes qui renvoient directement à l'épuration des danses modernes à laquelle ont largement contribué les Castles dans les années 1910 et qui a permis à la bourgeoisie de s'y adonner en toute respectabilité ${ }^{52}$. Effectivement, on y danse aussi bien la valse, un classique des plus appréciés, que le fox-trot, la rumba, la conga et le tango ${ }^{53}$. Mais on montre, toujours par l'image publicitaire, que la danse s'y pratique dans des attitudes irréprochables. En fait, la clé de la respectabilité se trouve dans la position des danseurs et dans une musique suffisamment sage pour leur permettre d'évoluer en maintenant cette position. Au début des années 1930, le professeur montréalais E. M. Vachon définit la bonne posture comme suit :

et Moyse Theatre, 13 au 15 décembre 1934, BAnQ, Collection programmes de théâtre; Current Events, 20 septembre 1935.

51 Témoignage de Myron Sutton recueilli et transcrit par John Gilmore dans Swinging in Paradise, p. 72.

52 Erenberg, Steppin'Out, p. 159-171; Don McDonagh, Dance Fever, New York, Random House, 1979, p. 27-29.

53 Valse : Matou botté, La Presse, 10 septembre 1932, p. 64, BVW, Fonds IVT, P101; Normandie Roof [cabaret de l'hôtel Mont-Royal], La Presse, 15 août 1939, p. 10. Fox-trot : Matou botté, La Presse, 10 septembre 1932, p. 64, BVW, Fonds IVT, P101. Rumba : Normandie Roof, La Presse, 15 août 1939, p. 10. Conga : Normandie Roof, La Presse, 15 août 1939, p. 10. Tango : Raymond Major, Souvenirs de St-Henri, [s. l. n. d.], CSSH, p. 21-22, BVW, Fonds IVT, P101; La Patrie, 11 octobre 1937, p. 17, et 3 février 1936, p. 16, BVW, Fonds IVT, P101; Normandie Roof, La Presse, 15 août 1939, p. 10. 


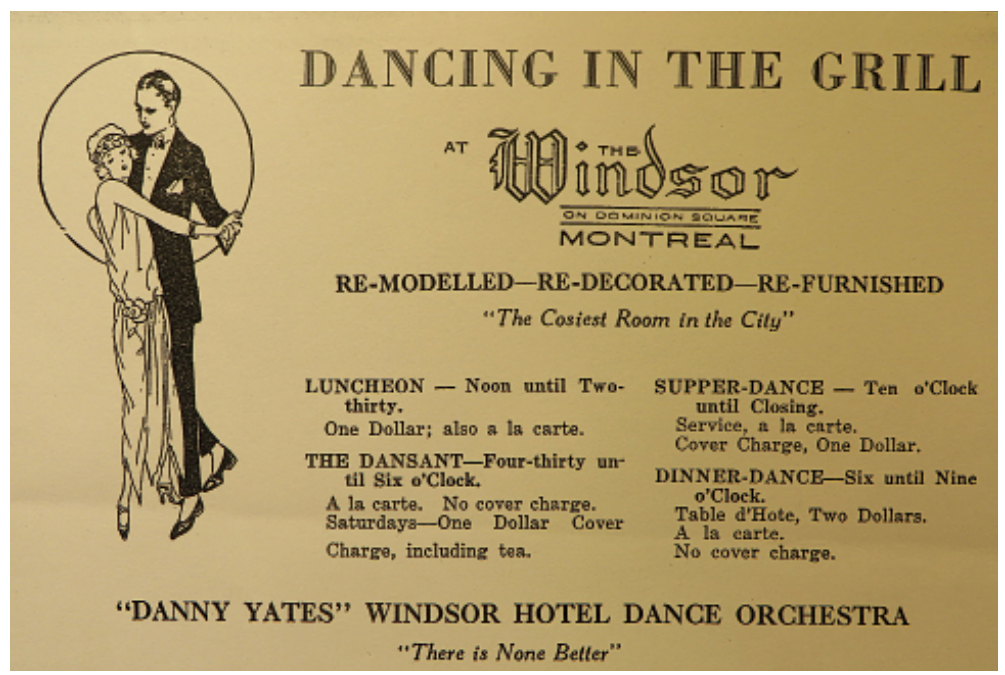

Figure 2 - Publicité de l'hôtel Windsor, Current Events, 26 octobre 1928, p. $8^{54}$.

«Le Monsieur place sa main droite en arrière au-dessus de la taille, et de sa main gauche soutient la main droite de sa partenaire en l'air, le corps rapproché mais non en face. La dame repose sa main gauche sur l'épaule du Monsieur, et il est préférable que chacun regarde audessus de l'épaule droite de leur partenaire pour raison hygiénique ${ }^{55}$." Qu'elles représentent un couple moderne, dont la femme est parfaitement habillée à la façon des flappers, ou un couple stylisé de danseurs de tango, les publicités de ces commerces réputés ne laissent place à aucune ambiguïté en reproduisant la position décrite par Vachon. Même si les danses modernes supposent que les corps soient

54 Publicité de l'hôtel Windsor, Current Events, 26 octobre 1928, p. 8; Publicité de l'hôtel Mont-Royal, Current Events, 20 septembre 1935; voir aussi publicité du Trocadero, Orpheum Theatre, 7 octobre 1928, AVM, Fonds Ægidius Fauteux, série 11, Programmes de spectacles, dossier BM001-11-D33; Publicité du Silver Slipper, Orpheum Theatre, 10 mars 1929, Ibid., dossier BM001-11-D34.

55 E. M. Vachon, Cours de danse social [sic] et populaire, [s. l. s. é.], 1931, p. 8; E. M. Vachon, Social and Popular Dancing Course, First Part, ("Translated from my french edition »), Montréal, La Patrie Print Co., 1935, p. 5. 
serrés l'un contre l'autre, les visages restent décalés, les regards ne se croisent pas, la main est haut placée sur le dos de la danseuse. Bref, la distance entre l'homme et la femme symboliquement maintenue prémunit de toute insinuation ou allégation de promiscuité.

Si le client souhaite aussi s'adonner au swing ou au jitterbug tout en restant dans un milieu destiné à une clientèle nantie et respectable, il peut se rendre Chez Maurice (1930-1931, SaintAlexandre; 1932-1940+, Sainte-Catherine Ouest, SAN) ${ }^{56}$. Pour lui, on a prévu un univers sélect avec des menus choisis, dont les prix s'échelonnent entre un dollar vingt-cinq et deux dollars, et un orchestre de swing composé de musiciens blancs qui jouent des musiques sur lesquelles les couples peuvent swinguer dans la position recommandée par Vachon, et comme l'indique la publicité.

Cette même publicité propose également des formes de sociabilité plutôt souples, avec " a pleasant atmosphere - cordial and friendly where you can rub elbows with the "Smart Set" ", et des salles "never overcrowded [...] to relax and mingle with your friends" (voir la figure 3). Chez Maurice entrouvre ainsi une fenêtre vers cet autre type de cabarets : ceux qui font une place à la sociabilité dite informelle, laquelle ouvre la porte à un éventail de comportements sociaux, depuis la simple détente jusqu'à la débauche.

\section{De la détente à la débauche}

Parce qu'elle se traduit par des interactions plus libres, plus spontanées, associées à des attitudes plus relâchées, la sociabilité informelle ménage une place à l'ambiguïté, celle-là même que les conventions et comportements exprimant l'idéal de la respectabilité bourgeoise de tradition victorienne présentés plus haut cherchent à éviter. La sociabilité informelle semble tout aussi éloignée des conventions et des comportements relatifs à l'idéal de respectabilité des milieux

56 Swing : Chez Maurice, His Majesty's, semaine du 3 février 1941, BAnQ, Collection programmes de danse. Jitterbug : Chez Maurice, La Presse, 20 janvier 1940, p. 43, BVW, Fonds IVT, P101. 


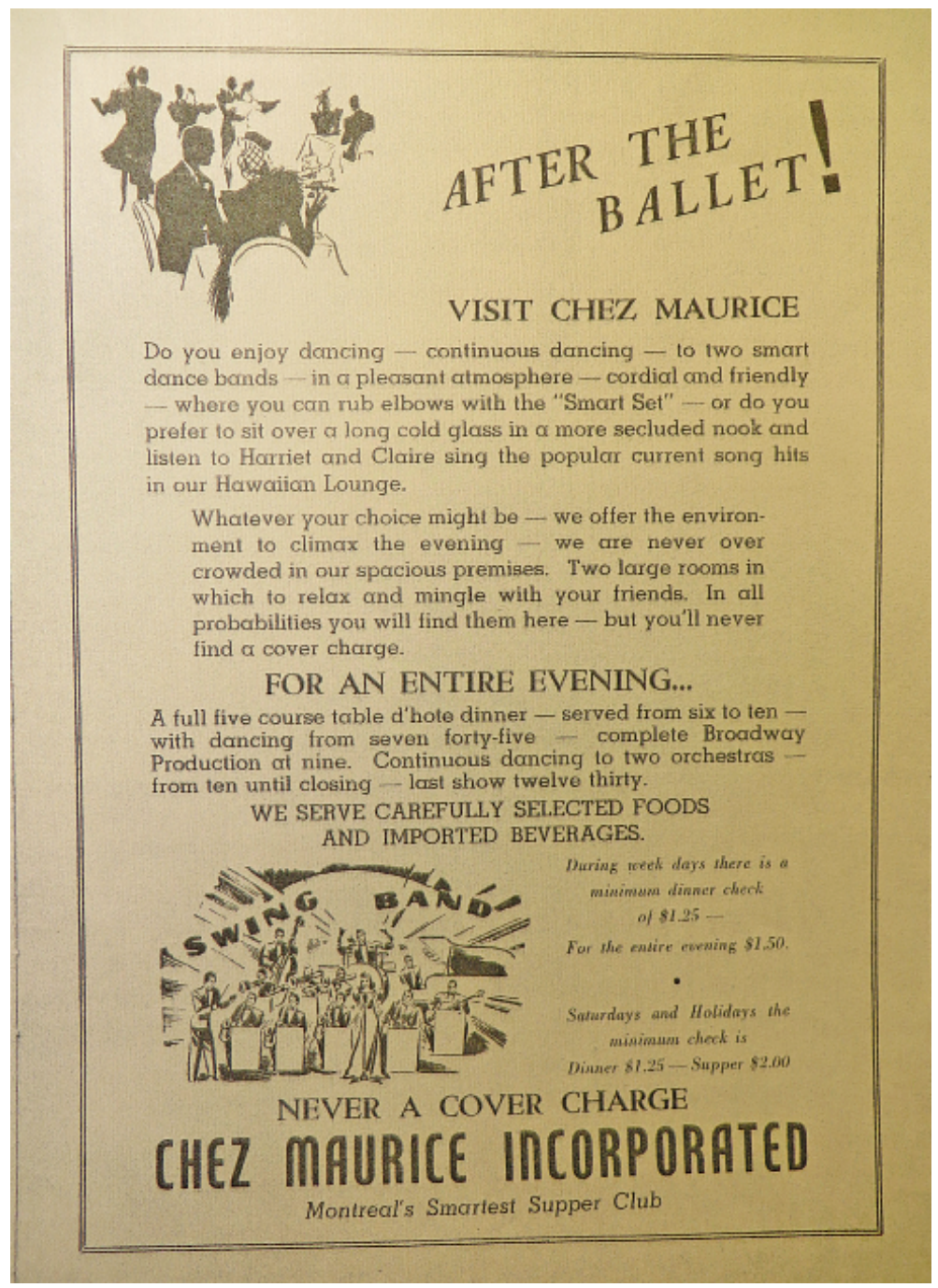

Figure 3 - Chez Maurice, His Majesty's Theatre, semaine du 3 février 1941 (BAnQ, Collection programmes de danse).

ouvriers, laquelle étant traditionnellement associée à la maternité, à la famille, au foyer et à la dignité se traduit notamment par le fait de se vêtir et de se loger proprement, de se tenir correctement et de 


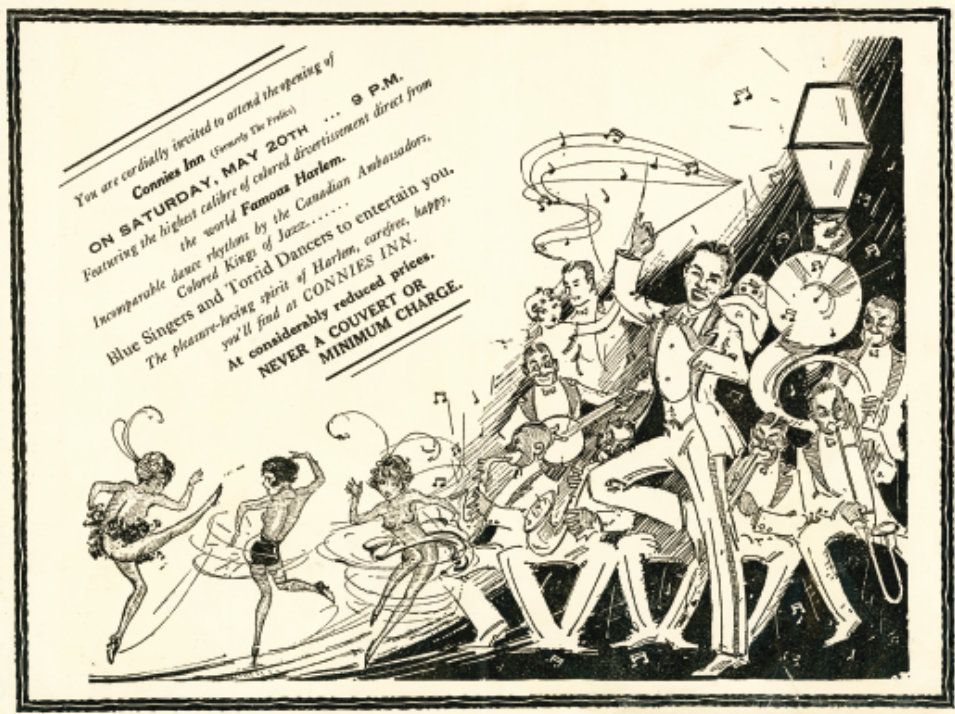

Figure 4 - Connie's Inn, 1933-1934 (CSC, Fonds Myron Sutton, P019, Spicilège 1, item 5, p. 2).

fréquenter les lieux qui ne remettent pas en cause le rôle prépondérant du foyer, de la famille et de la maternités ${ }^{5}$. Or s'habiller selon des modes qui peuvent faire douter de sa respectabilité et nécessiter des mises au point ${ }^{58}$, fréquenter des lieux et se mettre dans des situations telles qu'on semble prioriser l'indépendance et le plaisir, plutôt que la famille et la maternité, sont des illustrations de cette non-conformité aux conventions traditionnellement considérées comme garantes de la respectabilité. La sociabilité informelle est plus susceptible de permettre de tels comportements. C'est pourquoi elle devient source d'inquiétudes, fondées ou non, mais aux conséquences bien réelles pour les jeunes travailleuses en particulier; c'est aussi la raison pour laquelle elle est appréciée et recherchée.

${ }^{57}$ Myers, Caught: Montreal's Modern Girls and the Law, p. 77-84, 101; Bettina Bradbury, Familles ouvrières à Montréal : âge, genre et survie quotidienne pendant la phase d'industrialisation, Montréal, Éditions du Boréal, 1995, p. 125-129.

58 Peiss, Cheap Amusements, p. 98. 
Si le client souhaite des ambiances relâchées, des endroits où l'on est moins à cheval sur la bienséance, il peut compter sur des établissements plus ou moins flexibles en ce domaine, et plus ou moins abordables également. L'hôtel de LaSalle (1927-1940+, Drummond) est manifestement un établissement convenable de Saint-Antoine Nord, avec ses décors soignés et ses dîners à un dollar sans frais de couvert. Pourtant, en représentant des couples de danseurs, dont la main de l'homme est posée sur les hanches de la femme et non dans la partie supérieure de son dos, une publicité laisse supposer qu'on peut s'y permettre des attitudes plus sensuelles et une plus grande intimité59. Celui qui recherche des tarifs plus abordables, l'exotisme et une certaine liberté peut se tourner vers des établissements comme le Connie's Inn (1933-1934, Saint-Laurent, centre-ville Est). Le swing y est à l'honneur comme au cabaret Chez Maurice, mais plusieurs différences sont évidentes. Les "popular prices $^{60}$ " affichés deviennent, dans un livret publicitaire de 1933$1934^{61}$, de " considerably reduced prices. Never a couvert or Minimum Charge ". Le livret insiste sur l'ambiance débridée, "pleasure-loving spirit of Harlem, carefree, happy ", et promeut la culture populaire noire de Harlem en représentant un spectacle de musiciens et de danseuses noires presque nues (voir la figure 4). L'orchestre est celui de Myron Sutton, celui-là même qui mentionnait que son groupe ne jouait pas le même genre de musique que celle proposée dans les grands hôtels de Saint-Antoine Nord ${ }^{62}$. Les clients dessinés en train

59 His Majesty's Theatre, 6 janvier 1930, BAnQ, Collection programmes de théâtre.

${ }^{60}$ CSC, Fonds Myron Sutton, P019, Spicilège 1, item 6, publicité sans date.

${ }^{61}$ CSC, Fonds Myron Sutton, P019, Spicilège 1, item 5. L'illustration du livret est également disponible dans l'ouvrage de Marrelli, Stepping Out, p. 28-29.

62 Les Canadian Ambassadors font swinguer les clients de nombreux établissements : ceux du Terminal, dans Saint-Antoine Sud; ceux du Connie's Inn, du Chinese Paradise, du Club Hollywood, de la salle de danse Cartier, du Moonlight Gardens Café et du club Montmartre dans l'est du centre-ville; ceux de l'Auditorium Ballroom et du Venetian Village dans Saint-Antoine Nord (voir Centre d'archives, Université Concordia (ci-après CAC), Fonds John Gilmore, P004/A7.8, Research Material, photocopie annotée par John Gilmore des Spicilèges de Myron Sutton, 2 vol.). 


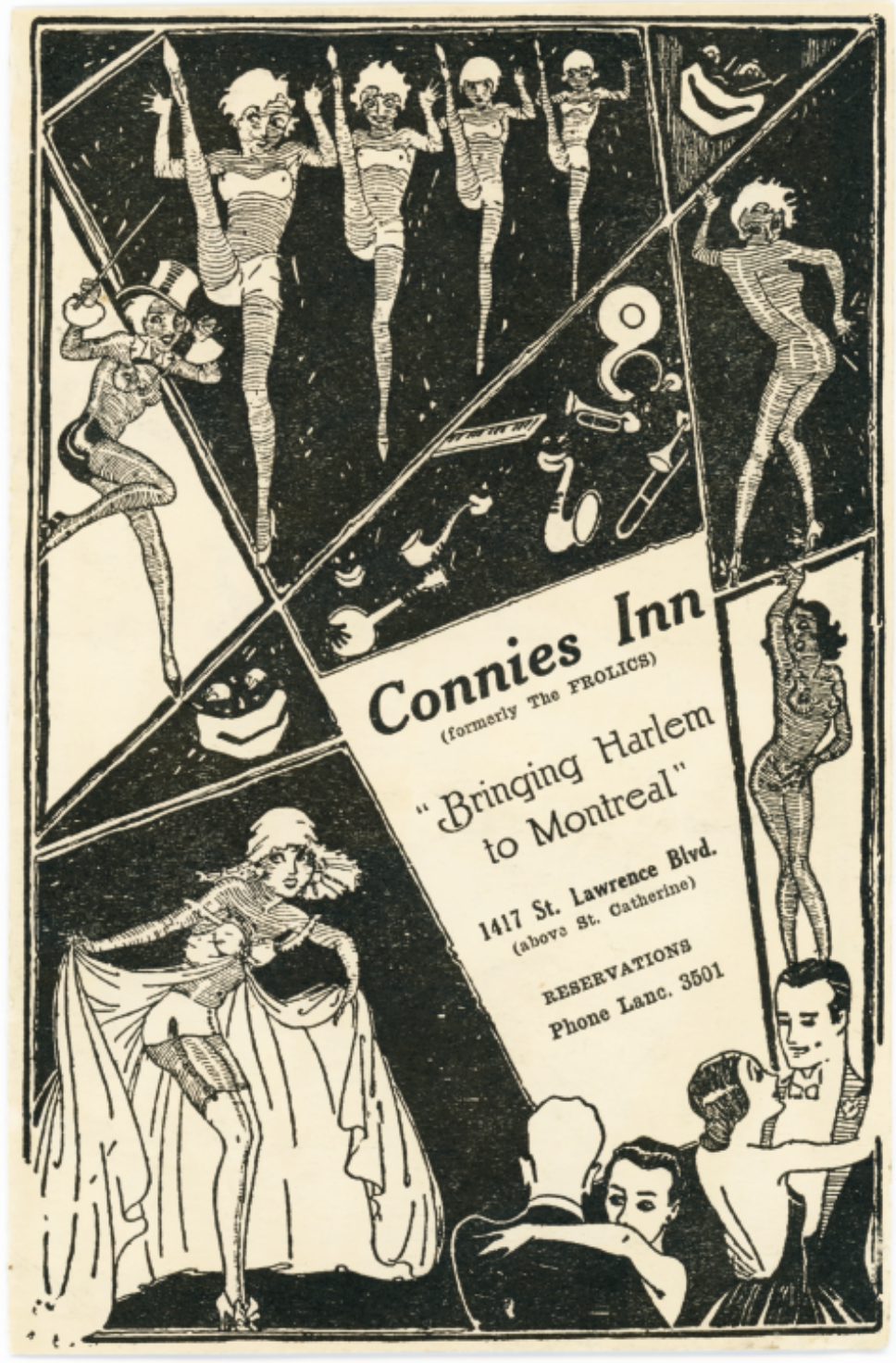

Figure 5 - Connie's Inn, 1933-1934 (CSC, Fonds Myron Sutton, P019, Spicilège 1, item 4, p. 1). 
de danser sont élégants, mais les exigences concernant l'attitude et la retenue ne sont pas comparables à celles valorisées chez Maurice. La position recommandée par l'étiquette n'est pas respectée et cède la place à une plus grande intimité entre les partenaires (voir la figure 5). Chez l'un des couples, la danseuse enlace son cavalier au niveau des épaules, de sorte que les bustes se collent tandis que les visages se trouvent par le fait même décalés. Chez l'autre, la main de l'homme est posée très bas sur le dos nu de la femme. Les deux partenaires se font face, se regardent et se sourient. Quant à la femme du couple qui paraît sur la page couverture, elle tourne carrément la tête à son partenaire de façon à se régaler du spectacle, ce qui, ailleurs, pourrait être un manque de savoir-vivre manifeste.

Si le succès du Connie's Inn est de courte durée (l'établissement ne survit manifestement pas à la fin de la prohibition américaine ou à la crise économique), d'autres cabarets comblent cet intérêt pour une culture et une liberté axées sur le plaisir de la musique et de la danse et non sur les conventions.

Certains cabarets de Saint-Antoine Sud donnent un accès privilégié au jazz, au swing et plus largement à la culture afro-américaine. Pour les amateurs de musique et de danse " jazzées ", y compris les gens de Saint-Antoine Nord, ces lieux apparaissent comme un cadre idéal pour accéder à des formes de sociabilité et à des façons de danser plus informelles, qui ne seraient pas considérées comme appropriées dans les commerces soucieux de répondre aux normes de respectabilité analysées précédemment. Le Terminal Club (1932-1940+, SaintAntoine Ouest) est un petit cabaret sans prétention, avec quelques danseuses qui présentent de brefs spectacles. Il attire une clientèle hétéroclite, surtout composée de blancs :

Very few colored people came in there, because it was an after-hours joint. When the big places closed uptown, people would come down and have a few more drinks. The gamblers would come down, the hustlers, the prominent people - it was a mixture. And we stayed 
late, till four o'clock sometimes. The doorman wouldn't let everyone in. He knew the clientele ${ }^{63}$.

Dans ce nightclub, les plus nantis qui viennent de quitter les établissements tenus par la loi de fermer à minuit (règlement municipal qui ne s'applique pas aux clubs) ${ }^{64}$ se mêlent au demi-monde. Ils révèlent ainsi la mobilité des individus selon le genre de détente qu'ils recherchent. Ici, la mixité sociale est garantie, bien que la clientèle soit triée sur le volet.

Les observations que fait Wilfred E. Israel au sujet de «Two Negro buffet parlors [qu'il nomme plus haut "cabarets"] [...] operated on St. Antoine St. at No. 1256 over a tavern and the other at No. 1323, over a Chinese laundry" (SAS) sont également instructives ${ }^{65}$. Israel s'intéresse à la vie de la communauté noire montréalaise et, dans ce contexte, aux comportements des habitués des lieux de danse de ce groupe. La démarche de l'étudiant en sociologie de McGill rejoint celle des sociologues de l'école de Chicago - Israel cite d'ailleurs les travaux d'Ernest Burgess ${ }^{66}$ - réputés comme " reproducing the dominant stereotypes of the day in their research ${ }^{67}$ » bien que cela soit très variable d'un chercheur à l'autre ${ }^{68}$. De fait, le témoignage n'est pas sans rappeler certains stéréotypes véhiculés à l'époque et dont quelques commerces jouent : le Terminal Club n’hésite pas à se présenter comme «Montreal's

63 Témoignage de Myron Sutton recueilli et transcrit par John Gilmore dans Swinging in Paradise, p. 80-82.

64 Règlement 776, Conseil de ville, Montréal, 1922, AVM, VM001-33-02-D057-776.

65 Wilfred Emmerson Israel, The Montreal Negro Community, mémoire de maîtrise (sociologie), Montréal, Université McGill, 1928, p. 189.

66 Sociologue de l'Université de Chicago dans les années 1920, Ernest Burgess a notamment émis la théorie selon laquelle les quartiers à proximité du centreville se dégradent au fur et à mesure que ce dernier grossit et sont délaissés par les habitants au profit d'autres zones résidentielles dès que ces derniers en ont les moyens (voir Yves Grafmeyer, "Ernest Burgess (1886-1966) » et "École de Chicago ", Encyclopedia Universalis, [CD-Rom], version 11, 2006).

67 Gotfrit, Dancing Back in the Jazz Age, p. 8.

${ }^{68}$ Steven C. Dubin, "The moral continuum of deviancy research: Chicago Sociologists and the Dance Hall ", Journal of Contemporary Ethnography, vol. 12, $\mathrm{n}^{\circ} 1$ (avril 1983), p. 75-94. 
Original Harlem Spot ${ }^{69}$ ". Selon Israel, " the cabaret or night club is the night life of this group [les Noirs] ${ }^{70} "$. En même temps, ses observations révèlent que la clientèle dépasse de beaucoup la communauté noire et reflète une mixité sociale, ethnique et géographique :

After 11 o'clock at night the patrons are to be observed drifting in the direction from their homes and from the auxiliary social centres in the sporting district. [...] By midnight these places are filled to their capacity. [...] The lady patrons of these cabarets are largely whites. These girls of the teen age and early twenties, some of whom are never seen in this district, except at night, come from all sections of the St-Antoine district. Above the hill has its representatives also ${ }^{71}$.

Cette clientèle mixte est attirée par ces établissements en raison des formes de sociabilité qu'ils autorisent. Israel explique :

Here the young are engaging in the prom[i]scuity phenomen as outlined by Burgess. Coming from different sections of the city, they enjoy the freedom and abandonment of their new contacts. There is an emotional excitement to these girls from the fact of being in stranger surroundings; the musical rhythm is most penetrating; the eating and drinking with the dark, soft-skinned male, supply that thrill and emotional release of unsatisfied wishes which she has sought so long. Live, eat, drink and be merry, for to-morrow I may die has become her philosophy for this brief but fascinating period ${ }^{72}$.

Cette sociabilité mettant à l'honneur la liberté est caractérisée par des termes fort connotés, en particulier promiscuity, abandonment, thrill et emotional release, mais le texte met l'accent sur une ambiance joyeuse plutôt qu'inquiétante ou immorale, comme l'indiquent les phrases suivantes : les instruments " grind out the sen[s]uous blue harmonies with a syncopation that sets the body in ready motion with

69 CSC, Fonds Alex Robertson, P023-C11, Alphabetical List, 18 juin 1938.

70 Israel, The Montreal Negro Community, p. 189.

71 Ibid., p. 189-190.

72 Ibid., p. 190. 
sympathetic vibrations ${ }^{73}$ "; la musique "is challenged by the hilarity and laughter of both male and female voices" et "the patriotic "God Save the King" at 5 o'clock in the morning brings the night's festivities to a happy conclusion ${ }^{74} "$. En faisant de l'hymne national la sonnerie du retour à la normale, Israel envoie un message fort : les libertés consenties par la nuit ont une fin; cette fin est consentie et ces libertés n’impliquent pas la perte du sens du devoir civique. Israel souligne aussi que chacun rentre chez soi (et non avec un partenaire) : "With the break up of these parties, the revellers are observed slowly wending their respective paths homeward. Often the young girls show facial signs of the glorious night that has been hers. Some walk after the pattern of the drunkard in their tired march home to rest for the frolic of to-morrow ${ }^{75}$. " Ce texte révèle la mobilité des clients selon leurs intérêts, notamment celui d'aller trouver ailleurs un type de musique et de danse, une ambiance et une liberté auxquels ils n'ont pas accès dans les commerces de leur propre quartier. Il permet aussi de constater que la liberté, la sociabilité informelle et la promiscuité ne constituent pas toujours des périls entraînant le déshonneur et la déchéance de la jeune fille - et ne sont pas toujours perçues comme tel, y compris par un jeune sociologue.

Cela dit, il n'est pas nécessaire d'aller dans certains cabarets de Saint-Antoine Sud ni même dans le Red light pour trouver des atmosphères plus permissives, des clientèles hétéroclites, voire des comportements ou des transactions qui flirtent avec l'illégalité. Dans SaintAntoine Nord, certains commerces accueillent une clientèle bigarrée et tolèrent des attitudes controversées tout en pratiquant des tarifs prohibitifs. C'est le cas du Savoy et du Bagdad Café qui sont mentionnés dans le témoignage de deux détectives de Chicago à la commission Coderre, résumé ici par Daniel Proulx :

3 Ibid., p. 189.

${ }^{74}$ Ibid., p. 190.

75 Ibid. 
Ainsi, au Sheik ${ }^{76}$, un cabaret rue Notre-Dame, " un orchestre noir exécute des airs de Jazz et les couples, encouragés par le gin et le whisky, font du "shimmy" et s'embrassent ". Même chose au Bagdad, situé en face de l'hôtel Mont-Royal et au Savoy, où un certain Tony Frank, le « Roi de la pègre » qui a trempé dans l'affaire du tunnel de la rue Ontario, côtoie le gratin du monde des affaires ${ }^{77}$.

Le Savoy, situé aux limites de Saint-Antoine Sud (1922-1993, Osborne, près du square Dominion), demande néanmoins un dollar pour le dîner dansant ${ }^{78}$. Pour sa part, le Bagdad Café ouvre en 1923 sur Peel, face à l'hôtel Mont-Royal, et n'hésite pas à profiter de sa situation pour exiger des frais de couvert de deux dollars pour le souper avec spectacle et danse au rythme des Garett's Irrestible Harmony $\mathrm{Men}^{79}$. Bien qu'il se vante d'être «the ultra-smart cafe where one can spend a pleasant evening amongst congenial surroundings and people; dancing on a good floor to a well balanced band ${ }^{80}$ ", il ferme ses portes dès 1924-1925, que ce soit à cause des frais de couvert excessifs (même pour Saint-Antoine Nord) ou des affaires qui s'y menaient, puisqu'on y trouvait également de la prostitution ${ }^{81}$. Dans ces commerces de luxe, l'intimité, le flirt, la clientèle bigarrée font manifestement partie du jeu. D'ailleurs, le shimmy est une danse très expressive qui, par l'accent qu'elle met sur les mouvements frénétiques du buste, soulève l'ire des moralistes. Ces commerces plus marginaux viennent élargir les formes de l'activité dansante récréative commerciale, tout comme les salles de danse.

${ }^{76}$ Cet établissement ne fait pas partie de notre répertoire, car nous n’avons pas pu le repérer.

77 Proulx, Le Red Light de Montréal, p. 18.

78 Princess Theatre, 7 février 1927, p. 10, BAnQ, Collection programmes de théâtre.

79 Orpheum Theatre, 3 décembre 1923, p. 11, AVM, Fonds Ægidius Fauteux, série 11, Programmes de spectacles, dossier BM001-11-D28.

${ }^{80}$ Current Events, 11 mai 1924.

81 Lévesque, La norme et les déviantes, p. 140. Remarque : le Bagdad Café est bien sur Peel, dans le district Saint-Georges, situé dans Saint-Antoine Nord, et où se pratiquait la prostitution clandestine, et non sur Notre-Dame, comme l'a noté Andrée Lévesque. 


\section{Les salles de danse}

Entre les deux guerres, le développement des salles de danse à Montréal est marqué par la stabilité des écoles et une croissance progressive mais constante des salles non associées à une école. Leur évolution n'est pas vraiment influencée ou concurrencée par celle des restaurants dansants ou des cabarets, car les salles de danse ont des fonctions tout à fait distinctes. En 1928, Percy A. Robert, un confrère d'Israel, décrit une salle du secteur Dufferin, manifestement le Jardin de danse ou l'Arcadia Ballroom qui lui succède en 1928, sur Bleury, juste au sud de Sainte-Catherine.

The only amusement place on the street [Bleury] is a Dance-Hall located just south of St. Catherine Street. It caters to the shop and factory girls and to their friends. Although there is a matron in charge, acquaintanceships are made very readily. A long bench, elevated from the floor, like the bench of a shoe-shine parlor, lines one wall. Here the girls sit waiting for a "pick-up". At one end of the hall a refreshment-parlor has been fitted up on a platform. At the opposite end there are checkrooms, wash-rooms and the orchestra.

Life here is accentuated, though not to the same degree as in the Dance-halls on the "Main. "Paled lights, the sob of the saxophone, the tinny sound of the jazz-band, and the girls'dresses of many hues create an artificiality approaching the life in the cabaret. The "sheiks" in carefully pressed wide trousers and meticullously parted "patentleather " hair glance over the bench on which the girls sit with cool apprisal, make their choices and drift onto the floor. And thus the evening passes.

When the dance is over more often than not the girls are deserted by their partners of the evening, and leave in two's and three's as they have come. Everyone pays for himself, and goes his own way.

This is the one colorful spot of the street ${ }^{82}$.

82 Robert, Dufferin District, p. 51. 
La salle de danse offre un produit simple : un lieu où danser avec des partenaires sans qu’il soit nécessaire de se présenter en couple. À lui seul, ce témoignage illustre le fait que cette offre détermine la configuration des lieux, les services offerts et qu'elle est intimement liée à un type de clientèle. D'abord la salle de danse, généralement sobre, donne accès aux infrastructures nécessaires pour danser : un orchestre, un vaste plancher de bois, des bancs de part et d'autre de la piste pour se reposer entre les danses et considérer les partenaires potentiels en face ${ }^{83}$. La salle peut également posséder des balcons, comme au New Auditorium ou au Palais d'or, éventuellement avec de petites tables ${ }^{84}$ qui ne sont pas destinées à la restauration cependant : seuls des rafraichissements peuvent être mis à la disposition des clients ${ }^{85}$. Le Palais de danse (1918-1919, Sainte-Catherine Est, centre-ville Est) fait exception en proposant le couvert, mais sur une autre plage horaire que la danse, soit de 11 h 30 à 14 h 30 et de 17 h 30 à 20 h $30^{86}$, alors que la danse se tient de 20 h 30 à 2 h $30^{87}$. La salle de danse concentre donc son offre de service sur la danse, ne proposant ni repas ni spectacle. En fait, savourer la prestation de l'orchestre et contempler les performances des autres danseurs, depuis les franges murales de la salle ou depuis les balcons, composent les seuls spectacles offerts à celui qui n'est pas en train de danser. Les annonces des salles de danse se font un devoir de présenter l'orchestre engagé, ce qui laisse supposer que la réputation et la qualité de l'orchestre peuvent contribuer à l'achalandage. Ainsi, le Stadium Ballroom (1929-1940+, hors du centre-ville, angle Ontario et de

83 Photographie de l'Auditorium, [1939?], CSC, Fonds Joe Bell, P010, Spicilège 2, p. 65, item B1.

${ }^{84}$ Conrad Poirier, Photographie du Palais d'or, 1944, BAnQ, P48-S1-P10534, [En ligne], [http://www.banq.qc.ca/collections/images/notice.html?id=06MP48S1S SOSSS0D0P10534].

85 Robert, Dufferin District., Conrad Poirier, Photographie du Palais d'or, 1944, BAnQ, P48-S1- P10534.

86 Princess Theatre, 9 avril 1918, AVM, Fonds Ægidius Fauteux, série 11, Programmes de spectacles, dossier BM001-11-D23.

87 Théatre canadien-français, 27 janvier 1919, Ibid., dossier BM001-11-D24. 
Lorimier) fait un coup d'éclat lorsqu'il organise, en 1934, une "Gigantic War of Jazz », événement qui réunit cinq orchestres : Geo. Sims and his Orchestra, Ken Large and his Orchestra, Henry Albert and the Rythm Kings, Hal Clark and his Broadway Collegians et, finalement, Myron Sutton and his Canadian Ambassadors-Colored Rythm and Hot Syncopation ${ }^{88}$. Ces orchestres permettent aux clients de s'adonner à diverses danses à la mode : fox-trot, valse, rumba, swing, one-step, big apple et shag ont été repérés dans des salles de danse. Notons que le botton swing, le charleston et les sets canadiens sont dansés dans des commerces montréalais qui restent indéterminés ${ }^{89}$.

Par son aménagement, la salle de danse est conçue pour permettre la rencontre d'un ou d'une partenaire avec qui on pourra danser. La dynamique décrite par Percy A. Robert rappelle étonnamment les bals du XIx siècle. Assises contre le mur, les jeunes filles attendent que les jeunes hommes viennent les inviter à danser, avec le risque de "faire tapisserie " si personne ne les invite. Les garçons, installés de l'autre côté de la salle ou dans ses coins, examinent les jeunes filles et font leur choix. Dans ce contexte, le groupe constitue un ensemble familier (au sein duquel la sociabilité, la complicité entre les membres sont déjà acquises) qui permet d'afficher une certaine allure, une confiance en soi et de garantir la sécurité de chacun ${ }^{90}$. Ici, les jeunes travailleuses viennent et repartent par deux ou par trois et sont surveillées par une matrone, garantie ultime de protection et de respectabilité en dépit des apparences qui pourraient être trompeuses. De même, le témoignage d'un jeune italien recueilli par Charles M. Bayley montre la force du groupe, soudé par son caractère indivisible :

The boys I go around with are from all parts of Italy. We haven't got much money but whoever has it pays. If one has a dollar he spends

\footnotetext{
${ }^{88}$ Gigantic War of Jazz, Stadium Ballroom, dimanche 8 avril [1934], CSC, Fonds Myron Sutton, P019, Spicilège 2, item 29.

89 Roquigny, Les plaisirs de la danse à Montréal, p. 515-521.

90 Peiss, Cheap Amusements, p. 113-114.
} 
it; if I have fifty cents I spend that - the one who hasn't any comes along with the rest. When we go in the car, each one dubs in three or four cents and we buy a gallon of gas - that takes us downtown and back. We generally go to dances $[\ldots]^{91}$.

En revanche, le groupe n'est pas un milieu dans lequel on recherche des partenaires puisqu'il n'est pas nécessairement mixte, comme l'indiquent ces exemples.

\section{Des clientèles variées}

Le fait que la rencontre et la constitution de couples de danseurs soit au cœur du fonctionnement des salles de danse a des conséquences sur la nature de la clientèle de ces établissements et sur les relations sociales liées à l'activité dansante. Dans ces deux domaines cependant, on observe une variété étonnante qui oblige à ne pas voir les salles de danse comme un bloc monolithique. Effectivement, ces commerces sont ouverts à une variété de clients, tant en raison de leurs caractéristiques sociales qu'à cause de leurs attentes en matière de sociabilité et de respectabilité.

Les jeunes, plus susceptibles d'être à la recherche de partenaires de danse - et éventuellement de partenaires de vie -, forment une clientèle toute trouvée pour ce type d'établissement. Cette donnée incite d'ailleurs la Ville de Montréal à préciser, dans le cadre d'un règlement sur les modalités d'ouverture et de fermeture des salles de danse, que les établissements ne doivent pas accepter " des enfants âgés de moins de seize ans ${ }^{92}$ ». Tamara Myers a néanmoins relevé des cas de jeunes filles de treize ans se rendant dans des lieux de divertissements nocturnes ${ }^{93}$. De même, si les salles de danse montréalaises

${ }^{91}$ Charles M. Bayley, The Social Structure of the Italian and Ukrainian Immigrant Communities in Montreal, 1935-1937, mémoire de maîtrise (sociologie), Montréal, Université McGill, 1939, p. 284.

92 Règlement 432, section 38 (a), Charte de la Ville de Montréal, 1931, p. 502, AVM.

93 Myers, Caught: Montreal's Modern Girls and the Law, p. 161. De même, selon Kathy Peiss, les dance halls new-yorkais sont fréquentés par des jeunes filles âgées de treize à vingt ans, jusqu'à ce qu'elles se fixent, et des jeunes hommes un peu plus âgés (voir Peiss, Cheap Amusements, p. 89). 
ne vendent pas d'alcool, mis à part l'Auditorium Dancing Hall (1913-1940+, Ontario, angle Bleury) qui aurait eu un permis ${ }^{94}$, la consommation illicite existe, comme le rapporte une jeune fille qui fréquente le Jardin de danse ${ }^{95}$.

À cette jeunesse peut se mêler une clientèle plus hétéroclite, notamment dans des salles du centre-ville Est et au taxi-dance hall (du moins selon ce que l'on sait de ce genre d'établissement), ce qui correspond bien au caractère hétérogène du quartier. Robert note que les salles de danse de Dufferin sont appréciées par " [the homeless man], as well as the younger people", "the foreigner seek [ing] companionship" et "the gang-boy [...] Here he meets the fringers of the demi-monde and is thus recruited into their ranks ${ }^{96}$ ". Ce type de clientèle se retrouve probablement aussi au Paradis Taxi-Dance Hall (1932, SainteCatherine Est, entre Sanguinet et Saint-Denis), étant donné la nature même de l'établissement et sa situation dans le Red Light. Paul G. Cressey, qui se distingue d'autres chercheurs de l'école de Chicago par sa volonté de ne pas porter de jugement de valeur et de décrire des comportements plutôt que de repérer des pathologies ${ }^{97}$, explique la particularité des taxi-dance halls dans une étude portant sur ceux de Chicago ${ }^{98}$ : seuls les hommes en sont clients; les jeunes femmes y sont payées à la commission pour danser grâce au "ticket-a-dance system, by which the girl received her pay on the basis of the number of tickets she could collect ${ }^{99}$ ». C'est le cas au Paradis Taxi-Dance Hall,

94 Gilmore, Swinging in Paradise, p. 95.

95 Myers, Caught: Montreal's Modern Girls and the Law, p. 160.

96 Robert, Dufferin District, p. 56-57.

${ }^{97}$ Dubin, "The moral continuum of deviancy research ", p. 85-89.

98 Paul G. Cressey, The Taxi-Dance Hall: A Sociological Study in Commercialized Recreation and City Life, Montclair, Patterson Smith, [1932] 1969, p. 181. Gotfrit a également mis en évidence l'intérêt de cette source. Elle précise notamment que Cressey avait été engagé comme "social scientist " par la Juvenile Protective Association of Chicago pour recueillir des informations sur les taxis-dance halls (Gotfrit, Dancing Back in the Jazz Age, p. 8-10).

99 Cressey, The Taxi-Dance Hall, p. 181. 
où le prix d'entrée est fixé à dix cents et la danse à cinq cents ${ }^{100}$. Ainsi, "like the taxi-driver with his cab, she is for public hire and is paid in proportion to the time spent and the services rendered ${ }^{101}$ ". Ce principe et ces tarifs flexibles semblent avoir une influence directe sur la clientèle, car si celle des salles de danse conventionnelles se compose majoritairement de jeunes travailleurs, celle des taxi-dance halls est beaucoup plus hétérogène : immigrants isolés; "footloose globetrotters " accoutumés à l'anonymat et à la mobilité; hommes jeunes ou mûrs, malheureux en mariage, divorcés ou veufs; handicapés et repris de justice; "slummers» incarnés non seulement par de jeunes hommes à la recherche de nouvelles sensations, mais aussi par des hommes plus âgés, cultivés, avec un certain statut social, mariés ou non, qui viennent profiter clandestinement d'une expérience peu conventionnelle à leurs yeux ${ }^{102}$.

Enfin, des personnes plus nanties constituent certainement une portion de la clientèle des salles de danse, notamment dans SaintAntoine Nord. Bien que, faute de témoignages, cela soit difficile à confirmer, plusieurs indices suggèrent cette hypothèse. D'abord, Saint-Antoine Nord, cœur des activités professionnelles et ludiques de la bourgeoisie, compte huit salles de danse entre 1919 et 1940 . Les établissements de ce quartier ne sont pas systématiquement et exclusivement destinés à la clientèle bourgeoise, mais cette dernière fait naturellement partie du bassin de clients potentiels. C'est évident dans le cas du Venetian Gardens (1918-1932, Sainte-Catherine Ouest) qui, se réclamant de sa proximité des hôtels Windsor, Ritz-Carlton et Mont-Royal, fixe son prix d'entrée à un dollar ${ }^{103}$. L'établissement troque cependant sa fonction de salle de danse pour celle de cabaret dès 1921, ce qui correspond peut-être mieux au profil de la clientèle visée, celle des grands hôtels. Ensuite, quatre de ces salles particu-

100 Le Paradis Taxi Dance Hall, 16 janvier 1932, CSC, Fonds Alex Robertson, P023-C10, Chronological List.

101 Cressey, The Taxi-Dance Hall, p. 5.

102 Ibid., p. 109-144.

103 Venetian Gardens, 4 octobre 1920, CSC, Fonds Alex Robertson, P023-C10. 
lièrement réputées sont associées à des écoles de danse, lesquelles sont au moins en partie fréquentées par la jeunesse bourgeoise, qui se doit de savoir danser : le Majestic Hall (1909-1926, Guy), l'Auditorium (1913-1940+, Ontario Ouest), le Stanley Hall (1898-1928, Stanley), que remplace Le Palais d'or (1928-1940+), et le Studio de danse Paramount (1930-1931, Peel). La jeunesse bourgeoise, qui connait bien ces écoles, est donc susceptible de profiter des séances libres aussi bien que d'autres clients.

\section{À chaque quartier son type de clientèle?}

A priori, on peut noter des différences entre les salles des trois secteurs, ce qui pourrait indiquer qu'on opère ainsi une sélection au sein de la clientèle. Outre le fait que la majorité des salles-écoles soient situées dans Saint-Antoine Nord, on remarque surtout que les prix d'entrée ne semblent pas gérés de la même façon dans les salles de danse de Saint-Antoine Nord et dans celles du centre-ville Est, si l'on se fie aux quelques cas pour lesquels on connaît les prix d'entrée. Dans Saint-Antoine Nord, seul le Venetian Village adopte la formule de la danse payante (cinq cents la danse, entrée gratuite ${ }^{104}$ ), sauf s'il s'agit d'une soirée spéciale ${ }^{105}$. Toutes les autres salles du quartier ont un prix d'entrée comme le Venetian Gardens : le Roseland Dance Hall (192, Place Phillips, cinquante cents en $\left.1921^{106}\right)$, le Palais d'or (1928-1940+, Stanley, entre trente-quatre et soixante-quinze cents selon le type de soirées ${ }^{107}$ ), l'Auditorium Ballroom (1913-1940+,

104 Venetian Village, 7 janvier 1933, CSC, Fonds Alex Robertson, P023-C10. Remarque : le Venetian Village est un établissement autre que le Venetian Gardens, mentionné par ailleurs.

105 Les tarifs sont fixés à vingt-cinq cents pour les femmes et trente-cinq cents pour les hommes, taxes et danse incluses, vestiaire pour dix cents et possibilité d'un rabais de dix cents avec coupon pour assister à une soirée spéciale avec Myron Sutton (voir CAC, Fonds John Gilmore, P004/A7.8, Spicilège de Myron Sutton, vol. 1, item 11b).

106 Roseland Dance Hall, 26 mai 1921, CSC, Fonds Alex Robertson, P023-C10.

107 Pour des exemples de danse à 40 et 65 cents, voir Montreal Daily Star, 2 janvier 1930 ; à 50 et 75 cents : Montreal Daily Star, 4 janvier 1930; à 40 cents : Montreal 
Ontario Ouest, cinquante cents pour les hommes et quarante pour les femmes ${ }^{108}$ ) et le Majestic Hall (1909-1936, Guy, trente à quarante cents selon le sexe et selon les annonces $\left.{ }^{109}\right)$. Au contraire, sauf événement spécial ${ }^{110}$, les quelques salles du centre-ville Est et hors centre-ville pour lesquelles nous connaissons les modes de tarification optent pour la danse payante, comme au parc Belmont, au Roseland Ballroom (1939-1940, Sainte-Catherine Est; entrée gratuite, prix de la danse non précisé ${ }^{111}$ ), au Paradis Taxi Dance Hall ${ }^{112}$ et au Verdun Boardwalk Dance Pavilion, qui proposent tous les deux l'entrée à dix cents et la danse à cinq cents ${ }^{113}$. Manifestement, les établissements des quartiers plus populaires souhaitent viser une clientèle la plus large possible en proposant un système de tarification flexible, permettant de dépenser pour une seule danse s’il le faut.

Malgré tout, on ne peut associer strictement les salles de danse de Saint-Antoine Nord à la clientèle bourgeoise et celles des autres quartiers à des clientèles populaires. D'une part, le cas des bourgeois et des jeunes filles de Saint-Antoine Nord se rendant dans les nightclubs de Saint-Antoine Sud nous incite à penser que les slummers de Cressey fréquentent probablement aussi les salles de danse montréalaises du centre-ville Est. D’autre part, les rares témoignages que nous avons

Daily Star : 12 avril 1935; à 34 cents : 17 avril 1935, 7 octobre 1936, p. 14, 9 avril 1937, p. 15; à 45 cents : 6 mars 1936, 14 avril 1937, dans BVW, Fonds IVT, P101. Exception : entrée à 25 cents avant 21 h lors d'une promotion (voir Standard, 14 janvier 1933, CSC, Fonds Alex Robertson, P023-C10).

108 Montreal Daily Star, 10 janvier 1930, BVW, Fonds IVT, P101.

109 Montreal Daily Star, 12 avril 1935, 2 novembre 1935 et 30 janvier 1936 respectivement, BVW, Fonds IVT, P101.

110 Le Festival de la musique au Stadium (1929-1940+, Ontario Est) coûte cinquante cents, plus dix cents pour le vestiaire (CSC, Fonds Myron Sutton, P019, Spicilège 2, items 29a et 29b).

111 CSC, Fonds Johnson, P088/2B, Music Industry, Box HA 695, Dossier Engagements-Contracts-Lists-Financial Record, 1939-1940.

112 Le Paradis Taxi Dance Hall, 16 janvier 1932, CSC, Fonds Alex Robertson, P023-C10.

113 Le Paradis Taxi Dance Hall, 16 janvier 1932, et Verdun Boardwalk Dance Pavilion, 4 juin 1938, CSC, Fonds Alex Robertson, P023-C10. 
trouvés permettent de constater que des jeunes gens de quartiers populaires situés au sud de Montréal Ouest, comme Saint-Henri et peut-être Saint-Antoine Sud, allaient au Palais d'or et à l'Auditorium Ballroom, situés dans Saint-Antoine Nord ${ }^{114}$. Ainsi, le jeune italien mentionné plus haut précise : "We [ses amis et lui] generally go to dances at the Stadium, Auditorium, Palais d'Or, anywhere and to the Cabarets - any one from Montreal West right up here ${ }^{115}$. " Pour sa part, Raymond Major note que les jeunes de Saint-Henri fréquentent aussi bien des restaurants dansants de leur propre quartier que des salles de danse situées en dehors du centre-ville (le Verdun Boardwalk Dance Pavilion et le Woodhall Dancehall (1916-1940, Gordon) ou dans Saint-Antoine Nord (le Palais d'or et l'Auditorium) ${ }^{116}$. Dans un sens comme dans l'autre, ces éléments montrent que les clients sont mobiles, ce qui ne peut que mener à une caractérisation nuancée des clientèles des salles de danse.

\section{Un éventail de comportements}

Cette caractérisation nuancée s'applique également à la nature des relations sociales qui s'établissent entre partenaires dans la salle de danse, depuis le partage éphémère et anonyme d'une danse à la séduction aux conséquences potentiellement problématiques, ce dernier aspect étant particulièrement connu ${ }^{117}$. Le Jardin de danse fait manifestement partie de ces salles considérées comme dangereuses. En témoigne une jeune fille enceinte qui affirme, devant la cour juvénile de Montréal, que l'alcool et la drogue y circulent et que l'établissement devrait faire l'objet d'une surveillance policière ${ }^{118}$. Cependant, Robert souligne, au sujet du même établissement,

\footnotetext{
${ }_{114}$ Major, Souvenirs de Saint-Henri, p. 22.

115 Bayley, The Social Structure of the Italian and Ukrainian Immigrant Communities, p. 284.

116 Major, Souvenirs de Saint-Henri, p. 22.

117 Myers, Caught: Montreal's Modern Girls and the Law; Strange, Toronto's Girl Problem; Peiss, Cheap Amusements; Gotfrit, Dancing Back in the Jazz Age.

118 Myers, Caught: Montreal's Modern Girls and the Law, p. 160-161.
} 
qu'une matrone est présente, que chacune paie pour elle (il ne fait pas mention d'échange de faveurs entre une jeune fille et un partenaire) et que la plupart des filles partent comme elles sont venues, sans partenaire. En évoquant ces trois éléments clés, Robert, comme Israel, présente des jeunes filles à la recherche de sensations, mais néanmoins respectables, plutôt que des charity girl ou casual prostitute sur la voie de la déchéance, ce qui incite à éviter toute généralisation hâtive.

De la même façon, d'autres témoignages concernant les salles où la danse est payante, plus susceptibles d'accueillir une clientèle populaire et même marginale, révèlent une réalité où le plaisir de la danse, entre amis ou entre inconnus, passe avant tout. Le témoignage, rapporté par Denyse Baillargeon, d'une dame ayant passé sa jeunesse à Verdun et allant danser au Verdun Boardwalk Dance Pavilion avec ses amis, laisse voir une forme de sociabilité conviviale, axée sur la bonne entente et la camaraderie, où l'informel et le plaisir ne remettent pas en question le respect de soi et de l'autre :

On dansait! Puis il y aurait pas un garçon qui nous aurait embrassées. C'était pas des cavaliers; c'était tous des copains. Quand on se rencontrait pour aller danser là, c'était pas comme aujourd'hui. [...] On avait du plaisir, mais c'était honnête. [Ces garçons] restaient alentour de chez nous. On restait tous dans le même quartier. On se rencontrait à l'hôtel Corona. On connaissait leur famille ${ }^{119}$.

Les souvenirs d'un homme concernant ses sorties à la salle de danse du parc Belmont avec ses amis sont pour leur part révélateurs de la prédominance de l'activité dansante sur toute sociabilité axée sur la séduction :

Nous allions danser au parc Belmont. C'était 10 cents la danse. Les filles étaient en rangs. Quand la musique arrêtait, nous allions nous chercher chacun une compagne. Les filles avaient des tickets et nous aussi. Je dansais et je ne disais même pas un mot. Je me

119 Baillargeon, Ménagères au temps de la Crise, p. 75-76. La dame interviewée ici est née à Verdun en 1908 et a travaillé dans une usine de cigarettes, jusqu’à son mariage en 1929. 
souviens des figures de danse, mais je serais en peine de me souvenir d'une figure de fille $e^{120}$.

C'est manifestement ce genre de réalité qui incite Charles Bayley à noter que " in the palatial pavilions and cabarets of Montreal's downtown, bright-light centre [...], relationships [...] are impersonal, secondary, segmental, anonymous ${ }^{121}$ ". D'ailleurs, le système de paiement à la danse renforce l'aspect uniquement dansant et temporaire du rapport établi puisque la personne peut très bien n'être là que pour une danse ou deux.

Par contre, au taxi-dance hall, où "young women and girls are paid to dance with all-comers, usually on a fifty-fifty commission basis ${ }^{122}$ ", des comportements très divers peuvent être observés entre les partenaires bien que la formule soit également celle de la danse payante et, qu'en conséquence, la durée de l'activité dansante puisse varier. Cela tient au fait que la jeune femme est, à titre de danseuse, à la disposition des messieurs. Elle ne peut ni refuser un client ni mettre fin à un partenariat de son propre chef ${ }^{123}$. Elle offre un service commercial, de sorte que la sociabilité du couple peut aussi bien être presque inexistante ou glisser vers la séduction instrumentale. Du côté des clients, la sociabilité peut aussi prendre ces deux tangentes selon les motifs pour lesquels ils se rendent dans ces établissements. Toujours selon Cressey, ces hommes fréquentent le taxi-dance hall pour des raisons utilitaires (apprendre à danser ou s'exercer pour un prix modique) ou plus romantiques (éprouver un sentiment de liberté anonyme, verser dans les jeux de séduction). Ces relations de séduction peuvent d'ailleurs être instrumentalisées par la danseuse, qui peut les favoriser ou du moins tirer profit de l'intérêt que lui porte un client pour lui faire dépenser le plus de tickets possible ${ }^{124}$.

${ }^{120}$ Marcelle Brisson et Suzanne Côté-Gauthier, Montréal de vive mémoire, 19001939, Montréal, Triptyque, 1994, p. 77. Sans précision sur le témoin.

121 Bayley, The Social Structure of the Italian and Ukrainian Immigrant Communities, p. 218.

122 Cressey, The Taxi-Dance Hall, p. 5.

123 Ibid., p. 5.

124 Cressey, The Taxi-Dance Hall, p. 109-144. 
Malgré leur unité apparente, les salles de danse ne constituent donc pas un ensemble homogène. Certes, elles offrent un service comparable et intéressent surtout une clientèle jeune à la recherche de partenaires de danse. Mais elles sont également susceptibles d'attirer des clientèles plus marginales ou plus aisées parce qu'elles offrent un milieu de pratique ou d'expérimentation différent, dépaysant même, et anonyme. Et si elles sont reconnues pour favoriser les rencontres et la promiscuité, elles accueillent, en fait, un éventail de comportements sociaux où la sociabilité informelle n'est pas nécessairement une atteinte à la respectabilité et peut même être entièrement évacuée au bénéfice de la danse, purement et simplement.

\section{Conclusion}

Comme le montre, chacun à leur façon, les sociologues cités tout au long de cet article, Cressey, Bayley, Robert et Israel, le danseur, la danseuse, est multiple et animé(e) dans sa pratique par toute une panoplie de préférences : passer toute la soirée avec la ou le même partenaire ou danser avec plusieurs; se rencontrer juste pour danser, socialiser, ou pour séduire; évoluer dans un univers plus ou moins respectable, modeste ou luxueux; préférer un milieu familier ou étranger à ses habitudes sociales et culturelles; rester dans son quartier ou aller voir ailleurs. La danse entre le consommateur et le commerce est enclenchée. Effectivement, toutes ces préférences qui peuvent être combinées différemment sont autant de profils potentiellement rentables pour le commerce, ce qui favorise le développement d'une variété d'établissements. Réciproquement, cette diversité incite le consommateur à définir et à réviser ses attentes, lesquelles peuvent changer au gré de ses préférences ou de ses besoins du moment. Ainsi, durant l'entre-deux-guerres, c'est une mer de possibilités qui s'offre au danseur même s’il pratique la danse récréative uniquement dans des espaces commerciaux. Si, d'une part, il peut choisir entre trois types d'établissements, d'autre part, le quartier dans lequel il souhaite se divertir, le prix qu'il est prêt à payer, 
le type de sociabilité qu'il recherche (formelle ou informelle, au sein ou à l'extérieur de son propre milieu social et culturel), le type d'ambiance et de musique et sa mobilité sont autant d'éléments qui entrent en considération dans le choix de l'établissement fréquenté. Consciemment ou non, les commerces semblent avoir saisi cette réalité. Dans certains cas, ils semblent tenter de la maîtriser en établissant implicitement une politique, un profil qui permet de cibler certaines clientèles, tant par la tarification que par les valeurs privilégiées dans le message publicitaire. C'est particulièrement évident pour les commerces qui souhaitent recevoir une clientèle visiblement respectable, telle qu'entendue dans la culture victorienne traditionnelle, qu'elle aime la danse sociale ou le swing, qu'elle soit nantie ou plus modeste. D'autres commerces aux tarifs abordables semblent plutôt miser sur le foisonnement, comptant sur la curiosité, le goût pour l'exotisme ou l'anonymat, pour attirer des clientèles plus hétéroclites mais aussi plus nombreuses. C'est notamment le cas de certaines salles de danse et de certains cabarets qui comptent, entre autres, sur les clientèles jeunes ou célibataires, mais pas exclusivement. L'étude de la pratique récréative commerciale de la danse doit donc prendre en considération non seulement les politiques commerciales, mais aussi les motifs et la mobilité des danseurs afin de révéler toute l'hétérogénéité de cette activité sociale et culturelle, au-delà des grandes tendances. 